\title{
Política de "extraordinarias circunstancias", mecanismo de crisis y de descontrol, frontera de actores móviles. Entre Ríos, 1830-1831
}

\author{
Policy of "extraordinary circumstances", mechanism of crisis and lack of control, \\ frontier of mobile actors. Entre Ríos, 1830-1831
}

\author{
Fabián Herrero \\ herrerofab@gmail.com \\ CONICET - Universidad de Buenos Aires, Argentina
}

Recepción: 17 Julio 2020

Aprobación: 08 Octubre 2020

Publicación: 13 Noviembre 2020

Cita sugerida: Herrero, F. (2020). Política de “extraordinarias circunstancias", mecanismo de crisis y de descontrol, frontera de actores móviles. Entre Ríos, 1830-1831. Anuario del Instituto de Historia Argentina, 20(2), e122. https://doi.org/10.24215/2314257Xe122

\begin{abstract}
Resumen: El presente trabajo analiza el caso del gobierno provisional de Pedro Barrenechea en Entre Ríos. Cuestiono la imagen historiográfica que lo presenta como una administración inactiva y sin propuesta ante las dificultades que presenta la escena entrerriana de los años de 1830. Muestro, por el contrario, que se trata de una administración con múltiples iniciativas de gobierno y con tomas de decisiones en áreas de poder, como el importante dominio de las comandancias militares. El archivo Urquiza, los documentos de gobierno y el congreso provincial del Archivo de Entre Ríos constituyen las principales fuentes.

Palabras clave: Gobierno, Entre Ríos, Siglo XIX, Política.

Abstract: This work analyzes the case of the provisional government of Pedro Barrenechea in Entre Ríos. I question the historiographical image that presents it as an inactive administration and without proposal in the face of the difficulties that the Entre Ríos scene of the 1830s presents. I present, on the contrary, that it is an administration with multiple government initiatives and decision-making in areas of power government, such as the important dominance of the military commanders. The Urquiza archive, government documents and the provincial congress of the Entre Ríos Archive are the main sources.
\end{abstract}

Keywords: Government, Entre Ríos, XIX century, Politics.

\section{INTRODUCCIÓN}

Hacia fines de 1830 y comienzos de 1831 Pedro Barrenechea asume como gobernador provisional de la provincia de Entre Ríos. Su administración forma parte de un cuadro de poder más extenso, que, de modo general, podríamos ubicar entre las décadas que se extienden entre la Revolución de Mayo y 1832, cuando asume Pascual Echague, quien permanece en el poder hasta comienzos de los años de 1840 (Ver Cuadro 1 en Anexo). La historiografía del período ha destacado dos cuestiones sobresalientes. En primer lugar, desde la perspectiva gubernamental o de poder provincial, se hace notar que, en ese extenso período, predominan gobiernos provisionales; esto es, son muy pocos los gobiernos propietarios elegidos por un proceso electoral 
que lleva a su designación por parte del congreso provincial. Se trata, además, de administraciones efímeras, con relativa o escasa duración en el tiempo, y que, a su vez, se mueven en un campo político, económico y social inestable, donde sobresalen nítidas aristas de crisis (Bosch, 1991; Martínez, 1910). El vocablo "extraordinario", en este cuadro de poder, es común en los papeles públicos del período y, particularmente, más común aún en los años que aquí analizamos. Tomamos como propio lo que señala al respecto Gabriela Tío Vallejo, quien, examinando el caso tucumano, ha denominado a este tipo de gobiernos como parte de lo que llama "republica extraordinaria": administraciones que, a su modo, asumen iniciativas de este carácter en un escenario de debilidad y de provisionalidad (Tío Vallejo, 2011).

En segundo lugar, vista la cuestión desde los actores, se ha destacado la presencia de "caudillejos" o "políticos menores". En el caso de Barrenechea, no sólo se han indicado estos últimos aspectos, sino que también se ha señalado que aquello estaba acompañado de otros: durante estos años ya era demasiado viejo y presentaba una personalidad débil (Bosch, 1991, pp. 117-133; Martínez, 1910: T. II, cap. VIII). Estos elementos, como se verá, son los que promocionan sus adversarios para descalificarlo.

Señalo mi perspectiva de análisis. En territorio entrerriano, durante la década de 1820, las rupturas institucionales, la crisis del erario público, los desbordes militares suponen una configuración compleja que, en términos generales, denomino mecanismo de crisis y de descontrol, y que se ha venido montando lentamente en esos años. Mecanismo que, cada gobierno de turno, ha tratado, a su modo, de cortar en seco, con escaso éxito.

La administración provisional de Barrenechea se presenta como un mirador ciertamente importante para examinar estos gobiernos débiles con escasa legitimidad, que emergen, desde una mirada a largo plazo, como pausas o esperas hacia un período de gobiernos estables. Un conjunto de problemas surge en este contexto alterado. ¿Cómo actúa un gobierno provisional sin legitimidad fuerte en medio de la formalización del Pacto Federal? ¿Qué hacer en relación con el problema militar de las comandancias departamentales, que son un actor importante en los "golpes de Estado"? ¿Qué hacer con los empleos creados por la reciente administración golpista? ¿Qué iniciativas prosperan en la economía o en el comercio, entre otras, en un gobierno débil?

En este marco es posible preguntarnos si la idea del gobierno de Barrenechea es proponerse (como señala la historiografía del período) como un gobierno de transito, o bien si pretende cortar en seco, tratando de dar algún tipo de respuesta a problemas tan urgentes como graves, con la intención de lograr que, finalmente, lo designen como un gobierno propietario. Creo que el segundo sendero es el que decide caminar Barrenechea. Para comprender mejor, entonces, este último esquema interpretativo, este trabajo analiza algunos aspectos relacionados con cuáles son sus estrategias, sus iniciativas y, sobre todo, sus alcances y límites.

En un segundo nivel de análisis, es posible examinar qué consecuencias tiene la escena de debilidad del gobierno provisional. La configuración del campo de poder va modificándose. Campo de poder, para emplear de modo muy libre la idea de Pierre Bourdieu, entendido como el espacio de juego dentro del cual los poseedores de capital, de diferentes tipos, luchan particularmente por el poder sobre el Estado. Los tipos de capital son diversos; entre otros, capital de fuerza física o de coerción (ejército, policía), capital económico, capital cultural, capital simbólico (Bourdieu, 1997, pp. 100-101).

En este marco, interesa separar tres dimensiones de análisis. 1) Un escenario de crisis: económica, militar, descontrol del territorio. Se trata de señalar, básicamente, qué tipos de problemas generan. 2) Las iniciativas de carácter institucional que intentan dar algún tipo de respuesta. Por este motivo, resulta pertinente examinar algunas leyes y reformas. 3) Finalmente, importa conocer algunas líneas vinculadas a las emociones, en cuan to hemos advertido una retórica dramática relacionada con las instituciones en los argumentos de esas leyes y órdenes de gobierno. Interesa saber, en este sentido, a quiénes y cómo se apela y, además, preguntarnos qué finalidad se busca.

En el interior de este cuadro de inestabilidad conspirativa casi permanente, dentro del campo del juego de la política de los actores, en el que se destacan Barrenechea, Pedro Espino, los comandantes militares, el 
Congreso provincial, los que resisten haciendo conspiraciones, se trazaron, a modo de hipótesis, una suerte de fronteras de actores que, al modificarse en el territorio y en el tiempo, se constituyen, en ese movimiento, en fronteras de actores móviles, pues sus movimientos resultan dinámicos, dentro de un tiempo que se acelera. Esta perspectiva puede resultar en verdad interesante para comprender cómo se movilizan los actores y cómo van modificando entonces las fracciones en pugna por el poder.

Los objetivos de este trabajo se dividen en tres secciones. En la primera, analizo el perfil del gobernador. En segundo lugar, examino distintos aspectos ligados a las acciones de gobierno, reformas militar y judicial, empleos, orden público, temas económicos. En tercer término, analizo el vínculo de la provincia con la Liga Federal y los movimientos conspirativos. Al final del trabajo, intento dar algún tipo de explicación sobre estos propósitos y me permito hacer alguna conjetura con la intención de explicar por qué este gobierno provisional, más allá de su intensa actividad, fracasa.

Este tipo de trabajo presenta, en verdad, varios tipos de riesgos. Se trata de eludir el circulo complaciente y empobrecedor de la historia que enumera hechos en una larga cadena temporal como si todos ellos presentaran la misma representatividad y relevancia histórica. Tampoco se intenta hacer ver que allí donde se postula, de modo general y machacón, un cuadro de caos sin sentido que no merece un mínimo examen, se podría ofrecer otro en el que sí parece que se presenta una serie indeterminada de hechos e iniciativas que mostrarían una escena iluminada por sucesos históricos no vistos suficientemente antes. Nuestra temática, en efecto, es tratar de examinar una administración que, por su excepcionalidad y su carácter provisional, no ha sido objeto de indagación con la seriedad que ella merece, en cuanto permite de alguna manera conocer el proceso político entrerriano en una perspectiva más compleja.

\section{Perfil de Pedro Barrenechea}

No hay referencias concretas sobre su origen. Los historiadores del período señalan que nace hacia fines del siglo XVIII en territorio entrerriano. En el período de la Revolución, mientras desarrolla actividades en la ganadería, en el espacio público se desempeña en las milicias en el pueblo de La Bajada y llega a convertirse en oficial. Integra, durante esa década, algunas de las empresas militares de carácter nacional: la expedición de Belgrano al Paraguay y luego, en el Sitio de Montevideo. Más tarde, participa en el Ejercito de los Andes y combate en las batallas de Chacabuco, Cancha Rayada y Maipú. Ocupa el cargo de Ayudante mayor del General Antonio González Balcarce en la segunda campaña al sur de Chile.

Tiene una activa participación en otros territorios provinciales. Interviene en la jurisdicción de La Rioja y en Mendoza. Diego, su hermano, entre los años 1817 y 1820, ocupa el cargo de Teniente Gobernador de La Rioja. Participa en el período de la revolución de Mariano Mendizábal. En 1820 vuelve a Entre Ríos. Forma parte de las fuerzas de Francisco Ramírez que combaten con José Artigas por la disputa del liderazgo federal en la región. Interviene en las batallas de Las Tunas y Mocoretá. Los especialistas han señalado que aquí es reconocido como un importante oficial de las fuerzas de Ramírez (Bosch, 1991, pp. 33-55).

En 1821 integra las fuerzas de Lucio Norberto Mansilla, quien combate contra las fuerzas de Ricardo López Jordán (padre) en Gená. Por sus actividades en este período es nombrado comandante militar de Concepción del Uruguay y es ascendido al grado de teniente coronel. En 1825 es nombrado gobernador en un breve período, como consecuencia del conflicto abierto entre Juan León Solas y López Jordán. Finalmente, Solas es nombrado de nuevo gobernador.

Este último, posteriormente, es acusado de pertenecer al partido unitario; por este motivo, se lo desplaza del poder en los meses finales de 1826. Vicente Zapata es nombrado gobernador. En este alterado escenario, Barrenechea integra las fuerzas de Solas que emprenden una revolución contra Zapata. El conflicto es resuelto a partir de la designación de Tomas García Zúñiga como gobernador y la detención de Solas y de Zapata. 
A fines de 1827, Zapata es designado gobernador. Esta vez, gobierna a partir de una alianza con Solas. En esta nueva administración, Barrenechea es nombrado comandante de Concepción del Uruguay, cargo que también ocupa cuando asume el poder Solas.

En noviembre de 1830, López Jordán toma el poder a partir de una revolución, con el apoyo de los hermanos Urquiza y otros militares. Piden la renuncia de Solas. La Legislatura lo reemplaza provisionalmente por Barrenechea, por una semana. Lo sustituye López Jordán. Gobierna por quince días, ya que no es reconocido por los gobiernos de Santa Fe y Buenos Aires. En ese marco, Lopez Jordan es reemplazado por Barrenechea quien ocupa el cargo de gobernador provisional, hasta la mitad de ese año. Es el período que examina el presente trabajo.

Se han destacado distintos episodios sobre su gobierno. Se ha hecho notar que su situación siempre fue inestable, en cuanto los jefes militares tejían sus propias alianzas con el fin de derrocarlo. Los hombres más destacados son su ministro Calixto Vera, cuya familia actúa en Santa Fe, y el Comandante de armas Pedro Espino (Bosch, 1991, pp. 75-97).

En suma, reúne algunos elementos habituales del capital simbólico de los actores notables del período: es un veterano de la lucha revolucionaria, un militar con fuerza de tropa; participa en territorios diferentes, no sólo en Entre Ríos; ha pasado por distintas crisis de poder, esto es, conoce de qué se trata la política de gobierno y su debilidad institucional. Llega a gobernador provisional luego de haber sido comandante militar de uno de los departamentos, cargo que, según los historiadores del período, tiene una importancia casi similar a la del primer mandatario.

\section{UN GOBIERNO EN BUSCA DE RESPUESTAS A DIFICULTADES URGENTES}

Señalo, rápidamente, las escenas quizás más relevantes de la administración provisional de Barrenechea. A la caída del gobierno "revolucionario" de López Jordán, que se extendió sólo un par de semanas, es designado como primer mandatario. Trata, como veremos a continuación, de reorganizar la provincia a partir de iniciativas de cambios de agentes y sanción de leyes y de reformas. Más tarde, pone fuera de la ley tanto a aquel líder revolucionario como a los hermanos Urquiza, y les confisca sus bienes. En enero de 1831 firma el Pacto Federal con Santa Fe y Buenos Aires, como una alternativa a las iniciativas de la Liga del Interior. En febrero de 1831, Entre Ríos es invadido por tropas de Lavalle y López Jordán, en la zona de Gualeguaychú. Apoyado por el flamante Inspector de Armas, Pedro Espino, Barrenechea los enfrenta con las armas. El hecho disruptivo encontró un punto de acuerdo con la firma de un armisticio y un indulto, que excluyó solamente a López Jordán y a Lavalle.

Se abre aquí un nuevo escenario que concluye con el desplazamiento de Barrenechea. Espino, luego de las batallas ganadas recientemente, se muestra como una de las principales espadas de la provincia; al mismo tiempo, Barrenechea se enferma y es presentado como un mandatario muy poco operativo a la hora de gobernar. En ese marco, en el mes de julio Espino arresta al gobernador delegado Vera (quien había asumido ese cargo por la enfermedad del gobernador provisional). Sin apoyos, Barrenechea renuncia a su cargo el 11 de julio. La Legislatura designa a Espino como gobernador provisional. ${ }^{1}$

Luego de abril, la frontera de actores móviles va modificándose: el "nosotros” cuenta con actores poco confiables y lentamente se configura un "ellos" que tiene a Espino como su cabeza visible. La Legislatura comunica al Poder Ejecutivo sobre el cese de las facultades extraordinarias acordadas en diciembre de $1830 .^{2}$ El gobernador delegado, José Ignacio Vera, rechaza los cargos de violación de la ley que en su contra le ha levantado la Legislatura. Habla de "injustas reconvenciones que se le hacen". 3

En suma, se advierte aquí cómo se levantan distintos escenarios. Al comenzar el gobierno de Barrenechea, se presenta una frontera de actores móviles, entre un "nosotros", los que integran el nuevo orden, y un "ellos", los "revolucionarios" derrotados. Pocas semanas después, cuando comienzan las voces de invasión 
y luego las acciones concretas, la frontera de actores móviles resulta, entonces, móvil, en cuanto ahora se separan un "nosotros", el gobierno y la Liga del litoral, y los "otros", los unitarios que conspiran. Derrotados a fines de marzo en el marco de un armisticio, la frontera de actores móviles vuelve a correrse, ahora entre un "nosotros", el gobierno, y un "ellos", que de modo ambiguo, en ocasiones, tiene como protagonistas a potenciales adversarios desde adentro de la administración: el ministro Vera (que a veces es también gobernador delegado) y Espino (el Inspector de Armas).

\section{Las primeras iniciativas}

Barrenechea, en efecto, debe enfrentar diversos órdenes de problemas: debe decidir qué hacer con los empleos designados por el gobierno "revolucionario de López Jordán". Pero también, con las deudas del Estado y los inconvenientes que se advierten en el comercio, sobre todo por el contrabando. A ellos hay que sumar las cuestiones de burocracia menor que resultan importantes a la hora de la concreción de los negocios y la participación del Estado como controlador, y qué medidas adoptar en relación con la falta de orden y de seguridad de los habitantes entrerrianos. Y debe establecer, al mismo tiempo, cómo será su participación en la Liga Federal.

Que la provincia en la que debe gobernar Barrenechea es altamente inestable lo prueba no sólo que, con la derogación del mando de López Jordán, aquel ordena a la Legislatura que todos los actos que emanen de aquella autoridad son de "una nulidad insubsanable", sino también la suspensión de elecciones programadas para diciembre hasta tanto se "calman las agitaciones de la Provincia y el Gobierno puede ponerse en contacto y comunicación con todos los pueblos de su mando". ${ }^{4}$

El tema de los empleos está directamente relacionado con la estructura institucional, pero también con actores, esto es, con las fuerzas de la propia "fracción" en el gobierno y la que se deja fuera de ella. De esta manera, las modificaciones o continuidades en materia de actores de una institución se relacionan al mismo tiempo con la apelación a emociones, en cuanto las instituciones no funcionan en abstracto, sino que lo hacen a través de personas (Lordon, 2018).

En el marco de este esquema interpretativo, respecto de los empleos se dan cuestiones típicas de momentos de anormalidad institucional. El gobierno de Barrenechea declara nulos los actos emanados del gobierno ilegal de Ricardo López Jordán, así como los nombramientos de ministros y demás empleados hechos por este. ${ }^{5}$ Hay un movimiento de restitución de empleos que habían sido declarados caducos por el gobierno "revolucionario". Es el caso del Capitán del Puerto y comandante del Resguardo D. Ciriaco Quintana, quien fue, señala el gobernador, "injustamente despojado de su empleo y por una autoridad ilegítima". Por este motivo, "queda repuesto en ellos, sin que pueda perjudicarle en manera alguna la momentánea separación que ha sufrido". 6

En el momento de los cambios de gobierno se presentan abandonos de empleos. El gobierno declara cesantes de sus tareas a "los prófugos" de Concepción del Uruguay D. José J. Sagastume y el interventor D. Juan J. Irigoyen. Estos "abandonaron sus empleos, fugando de aquel Pueblo en el mes anterior" y lo hicieron "sin que el Gobierno tenga noticias de quienes los hayan sustituido, por consecuencia se declara que aquellos individuos han cesado en sus empleos". ${ }^{7}$ Lo que se advierte es el desorden que genera la anormalidad institucional: el empleado de la aduana no obedece las órdenes emanadas por el gobierno provincial.

Al mismo tiempo, se producen movimientos que no se justifican. Por un decreto, el Poder Ejecutivo destituye al Administrador de Nogoyá. No da detalles: sólo dice que "el Gobierno tiene motivos muy poderosos" para separar "del empleo de Administrador del pueblo de Nogoyá al que hoy desempeña Luciano Godoy". ${ }^{8}$ En el mismo decreto se afirma que el Gobierno ha nombrado comandantes interinos de Nogoyá y La Matanza al Capitán D. Francisco Amarillo y al Capitán D. Mariano Sosa, respectivamente, con lo que cesaban en sus cargos D. Ramón Ascua y D. Hilario Campos. ${ }^{9}$ 
Anormalidad institucional, puesta en obra de nuevas comunicaciones institucionales con los pueblos de la provincia, ausencia de calma social, apelaciones a valores mezclados con sentimientos generales como "injusticia", "indiferencia", pero también una retórica vacía que apunta a justificar iniciativas sin dar motivos precisos, surgen a primera vista como los principales rasgos que prevalecen en el comienzo de la administración provisional de Barrenechea.

\section{Temas del erario: sobre deudas y comercio}

En materia económica, el gobierno trata de dar algún tipo de respuesta a temas de deuda y comercio. Esas respuestas, o intentos de ellas, surgen de una necesidad: introducir en el territorio entrerriano un principio de orden y de clasificación, con el fin de contar con reglas claras en el dominio institucional. En ellas también puede advertirse cómo los argumentos y las justificaciones vinculan lo institucional con apelaciones a las emociones, en cuanto las instituciones, como señalamos más arriba, no funcionan en abstracto, sino que con sujetos, y estos no actúan aisladamente sino vinculados a aquellas.

Estado con deudas. Las primeras medidas en materia económica tienden, en efecto, a recaudar fondos en un escenario alterado por la crisis de la provincia y por la reciente revolución de López Jordán. La enumeración de estas medidas durante las primeras semanas del mandato de Barrenechea es un claro botón de muestra.

El Gobierno, de este modo, usando de "las facultades extraordinarias de que se halla investido", teniendo en cuenta la "complicación de las circunstancias", que exigen "no solamente grandes y extraordinarios sacrificios, sino el que se suspende el curso ordinario de los negocios", ha acordado que el Ministro de Hacienda "suspenda por ahora toda clase de descuentos, sin excepción de los "suplimistas" en la última contribución impuesta por D. Ricardo López Jordán”, y, además, "proceda con la rapidez posible a cobrar todos los créditos pendientes que tenga el Estado"; al mismo tiempo, debe prevenir a los deudores que el día de mañana deben, precisamente, "cubrir sus deudas". En tono dramático se aclara que "de esto pende realmente la salud pública y la salvación del país”. ${ }^{10}$

El ministro, en la misma línea, previno a todos los deudores del Estado, que en el término de una hora entreguen en cajas las cantidades que adeuden, en inteligencia que, no verificándolo, el Gobierno adoptará las medidas que corresponden, para hacer cumplir una medida de que depende la salud pública y salvación del país. ${ }^{11}$

Para conseguir este objetivo, posteriormente, se insiste en la necesidad de buscar elementos y recursos. Un Acuerdo ordena al ministro que "mientras duren las presentes circunstancias, y los grandes peligros que amenazan la provincia”, efectivamente, se "suspenda todo descuento, y ordene el pago íntegro de los derechos de introducción y extracción, en el acto mismo que los verifiquen los particulares". ${ }^{12}$

El gobernador Barrenechea le señala al ministro que suspenda cualquier pago que tenga el Estado y "exija el pago de los créditos pendientes a favor del Estado", "dando cuenta de los que se resistan al cumplimiento de esta resolución" y se tomen las medidas del caso debido a la urgencia de las circunstancias. ${ }^{13}$

Se establece que aquellos con los que el Estado tenga deuda cuentan con un plazo de seis meses para presentar los documentos correspondientes. Un plazo mayor reciben los que se hallan fuera de la provincia. El gobierno luego clasifica y reconoce a aquellos que lo merecen y se compromete a pagar. Otro artículo aclara que el pago se hará según la cantidad a la que ascienda la deuda total. ${ }^{14}$

Se trata de medidas habituales en momentos de crisis, cuando un Estado pretende retomar la iniciativa, ordena, obliga, exige; iniciativa que está acompañada de una retórica dramática, que busca conmover socialmente: se apela a que es el último y único recurso para salvar la existencia misma de la provincia.

Sobre el comercio. Se intenta dar un orden para su buen funcionamiento, con el fin de que los comerciantes logren obtener beneficio, pero también para que el Estado pueda recaudar. Como veremos, se advierten problemas de recursos y de orden institucional para recaudar, y se busca una solución inmediata, 
que muestra movimientos de improvisación. En efecto, aquí también se advierte el Estado que retoma iniciativa con una retórica dramática que busca conmover socialmente

Instruido el Gobierno de que muchas personas se embarcan diariamente sin la correspondiente licencia con grave perjuicio y menoscabo de los intereses del Estado, que en el ramo de papel sellado sufren un desfalco considerable, en circunstancias en que el país reclama imperiosamente grandes y abundantes recursos para su salvación, ha acordado colocar una persona que merezca su confianza, para que en el mismo puerto venda el papel sellado a todos aquellos que por un olvido, precipitación en su viaje o ignorancia, no hayan recabado de la Comandancia General la competente licencia, y que allí mismo el Capitán del Puerto y Comandante del Resguardo les extienda el correspondiente pase. ${ }^{15}$

La solución es la designación de un empleado entre quienes "no sean personas sospechosas o deudores al Estado”. Con estas credenciales, designan a D. Pedro Otaño, quien, a los ojos de la nueva administración, reúne las "calidades necesarias para el desempeño", con el objetivo que se "encargue de la indicada renta, con el goce de 4 pesos, concedidos a los de su clase". En la orden se pide que el ministro actúe con "urgencia" para poner en obra dicho nombramiento. ${ }^{16}$ Se nota aquí la escena de un Estado que no tiene control, y una administración que intenta dar algún tipo de respuesta con el objetivo de "normalizar" la situación del comercio, pero también el de recaudar.

Se sanciona, con un propósito similar, un decreto del gobierno que reglamenta la Hacienda en cuanto a la entrada y descarga de buques. Se trata de "medidas parciales hasta que preparen el plan general que debe oportunamente levantarse". Es un control sobre los buques cuando están en los puertos: quiénes son los patrones o capitanes, qué papeles llevan, qué piezas van a bajar y de qué se trata, qué pasa si no presentan estas "piezas van a bajar" en un breve plazo, etc. ${ }^{17}$

El Gobierno le señala al Ministerio que, con el fin de arreglar y aumentar las rentas públicas, resulta sumamente necesario "evitar el fraude y contrabando tan generalizado en todas partes". ${ }^{18}$ Para ello, elabora un decreto.

Los primeros artículos ( 1 a 4 ) están dedicados a advertir sobre situaciones habituales: no se puede conducir cargamento sin licencia, bajo pena de decomisado de las mercaderías en caso de no cumplir con ello; dispone medidas sobre su descarga al puerto. Lo que la embarcación descargue o cargue debe figurar en las guías correspondientes ( 7 a 9 ).

Los artículos 5 y 6 aluden a la figura del delator. Por el primero, "todo guarda, todo ciudadano, todo habitante en la provincia tiene derecho a delatar el contrabando, y a la obtención a la mitad de lo que se pilla en esta clase". Y por el segundo, se establece que el "Gobierno guardará a los delatores un secreto inviolable y será inexorable y religioso en su cumplimiento". A su vez, el artículo 11 señala que "todo individuo tiene derecho de aprehender el contrabando que se encuentre y conducirlo a la aduana, o a algún punto de seguridad, donde esté custodiado hasta el siguiente día en que dará inmediatamente aviso al Gobierno o al Ministerio de Hacienda, y en los demás Pueblos a los Administradores y Alcaldes Mayores". Se puede suponer aquí una situación que se extiende a otras. Me refiero a que la idea del delator va unida no sólo a la de un habitante responsable con la comunidad, al que se le pide colaborar con el orden y las reglas, sino que hay detrás una recompensa por esa delación. Aparece como una especie de derecho, como es el caso del botín después de algún conflicto bélico, como hace tiempo Raúl Fradkin, lo viene mostrando con particular sutileza (Fradkin, 2006).

El tema de los castigos parece cumplir los requisitos habituales, en cuanto se los establece de acuerdo con la reiteración de los delitos. El artículo 10 señala que "el que contraviniese el artículo anterior pagará por primera vez irremediablemente la multa de mil pesos y sufrirá tres meses de prisión, por la segunda dos mil y seis meses del mismo castigo, y por la tercera después de igual tiempo de prisión, no podrá en lo sucesivo traficar en los Puertos de esta provincia con buque a su cargo". En el caso de Córdoba, en un período relativamente similar, hemos observado cómo se sanciona una ley que protege, de algún modo, a los comerciantes que 
hacen contrabando, pues deben cometer varias veces el mismo delito para que reciban algún tipo de sanción (Herrero, 2007).

Las iniciativas hacia las deudas del Estado y el comercio aluden a cuestiones relativamente elementales en cualquier administración, sobre todo en momentos de crisis (Tedeschi, 2020). Estos temas, que están ligados a la recaudación por parte de aquella, cumplen una función particular. "La concentración de la fuerza física (fuerzas militares o de policía) pasa por la instauración de una fiscalidad eficiente" (Bourdieu, 1997, p. 101). Por el contrario, su falta de eficacia en el dominio de las fuerzas, más otras crisis sociales o económicas, son potenciales motivos de conspiraciones o revoluciones (Todd, 2000). En este preciso sentido, se comprende mejor por qué una parte de la nueva invasión de los unitarios y la posterior usurpación del poder por parte de Espino tuvieron, sin duda, un escenario favorable para sus propósitos. Y, por otra parte, por qué el gobierno provisional y "extraordinario" de Barrenechea debía con urgencia realizar las reformas e iniciativas que consideraba de utilidad para dar respuestas a dificultades graves.

\section{Reforma militar y judicial ¿una reforma del "estado provincial”?}

El 14 de enero de 1831, el gobernador Barrenechea envía una nota al Congreso provincial para que se cree un cargo de Inspector de Armas de la provincia, que tiene como fin cortar el nudo del problema regional que desde hace años divide a la provincia de Entre Ríos. A propósito de la nota del gobernador, el Congreso provincial elabora dos leyes en torno al orden militar y de justicia. Como todo nuevo ordenamiento legal, se presenta un argumento inicial que justifica su sanción explicando los inconvenientes que se registraron hasta aquí.

El origen de los inmensos males que hasta hoy han afligido al País no era otra que (...) la impropia división de una misma provincia en dos mitades, cuya total independencia reducía a nulidad la acción del gobierno y que tanto los Comandantes Generales del Uruguay como la multitud de comandantes subalternos absorbían las rentas del Estado de tal modo que no ha sido posible sobreponerse a la miseria a que el erario público era reducido para esta causa. ${ }^{19}$

Como se ve, son problemas de autoridad y de erario público los dos aspectos que justifican su elaboración. En la opinión del Ejecutivo, la provincia no dispone de un poder central provincial fuerte en la misma medida en la que los comandantes militares, uno en Paraná y otro en Concepción del Uruguay, disponen de un poder que hace que ella divida el suyo en dos. Hecho que produjo, lo insinúa, pero no lo señala directamente, los golpes institucionales de toda la década. Destaca, además, que la actual distribución militar en la provincia permite una "multitud" de comandantes subalternos que ocasionan gastos que el erario provincial no puede solventar. Aquí se hace referencia, aunque tampoco de modo explícito, a los problemas de falta de fondos del erario a la hora de pagar sueldos a los militares, dificultad que fue uno de los tantos motivos de las irrupciones militares.

En este marco, la primera ley modifica radicalmente el orden militar establecido hasta aquí. El artículo 1 crea el "empleo de Inspector y Comandante General de Armas", destacando que fue propuesto por el gobierno de la provincia. El segundo artículo establece que "quedan suprimidas las dos comandancias Generales y las subalternas de la provincia", y lo disponen también en lo "relativo a la división del territorio en lo civil". De este modo, destacan que así todo queda conforme con el "apéndice $1^{\circ}$ del Estatuto Provisorio Constitucional".

Estos cambios generan un nuevo escenario en el orden de la justicia. Por este motivo, se aclara en el artículo 3 que en breve se "arreglará" la mejor forma para "proceder en los Juicios civiles de mayor cuantía”, y en los "criminales en la parte que por apelación corresponda a dichos comandantes generales", los cuales constan en varios artículos específicos del Estatuto Constitucional. ${ }^{20}$

En una segunda ley, el Congreso provincial establece por el artículo 1 la creación en la "Capital de unTribunal Superior de Apelación”, que será integrado por un presidente y dos vocales nombrados por el 
Poder Ejecutivo; “su duración será la del Gobierno" y el sueldo se establecerá según se encuentren las rentas de la provincia, a la vez que se indican particularmente los artículos del Estatuto de la provincia que al respecto serán derogados. ${ }^{21} \mathrm{El}$ articulo 2 explica el tema de los juramentos, en el 3 se especifican otros artículos derogados en relación con los Comandantes generales. En el artículo 4, se señala que los "Alcaldes Mayores de los cinco pueblos” (se refiere a Paraná, Nogoyá, Gualeguay, Gualeguaychú y Concepción del Uruguay) son considerados "iguales en sus facultades y dependen de ellos los Alcaldes de Cuartel y de Hermandad comprendidos en sus respectivas jurisdicciones". En los artículos 7 y 8 se dan otros pormenores acerca del tema de la justicia sobre estos actores. En el 5 y el 6 se establece qué hacer en casos de demandas de menor cuantía y otros artículos derogados en el Estatuto constitucional. Se alude, además, a una nueva figura legal que "manda" en los pueblos. En el artículo 9 se sostiene que ellos serán "mandados por los alcaldes mayores", a quienes se les "conferirán las facultades de comandantes y jueces de policía”; se aclara, además, que se les asignará "un sueldo, que se acordará a la brevedad posible, y con concepto al Estado de la provincia”.

En suma, hay cambios sustanciales, no sólo en la distribución de poder de los militares en la provincia sino también en el campo de la justicia. Todos ellos van acompañados de una modificación notable del estatuto constitucional. Se trata de uno de los tantos intentos de cambio en la trama judicial desde la sanción del Estatuto hasta su reforma en 1849 (Pressel, 2020).

En este cuadro de renovación y cambio, Pedro Espino es nombrado como Inspector de Armas de la provincia. Se trata de un empleo "en propiedad", esto es, no es provisional ni transitorio. Tiene un salario acorde a las demandas de sus tareas. Por este motivo, en el papel público que lo anuncia se aclara que "no puede ser otro que el de mil quinientos pesos anuales", no sólo por "la decencia y rango que por su elevada posición debe sostener precisa e indispensablemente", sino y, sobre todo, porque su empleo "no tiene residencia fija, y en su continua movilidad, debe originarles gastos de la mayor consideración". ${ }^{22}$ Este esquema argumentativo va acompañado de una retórica que apela a la emoción. Cuando se lo nombra, se señala especialmente: "este valiente oficial". Se destaca, además, que el nuevo cargo es un premio a su "mérito singular", por haber vencido a los "revolucionarios" de diciembre. Espino, en efecto, "supo salvar a la patria y arrancarla de las garras de un traidor, que con sus engaños se había apoderado de ella". ${ }^{23}$ Todo esto tiene, en verdad, su parte de farsa, en la medida en que se omite que uno de los comandantes que acompañaron a López Jordán en su acceso al poder fue justamente Espino.

A modo de hipótesis bien podría decirse que, con su designación, se le da a Espino, una de las tendencias vencidas en diciembre, una importante porción de poder: el mando militar de la provincia. El recreado escenario político no se organiza en torno a una fuerza nueva, sino que se trata de una alianza entre sectores distintos que mezcla recientes revolucionarios y veteranos de la política provincial.

\section{VÍNCUlos CON LA Liga FEDERAL, FRONTERA DE ACTORES Y MOVIMIENTOS CONSPIRATIVO el Pacto Federal}

\section{El Pacto Federal}

En este período del gobierno de Barrenechea, diciembre 1830 y julio 1831, uno de los ejes temáticos es su vinculación con la Liga Federal. Por este motivo, en la sesión del último día de 1830 el Congreso provincial acepta la renuncia del diputado secretario Antonio Crespo, lo designa diputado para la reunión de las provincias en la ciudad de Santa Fe y le asigna un sueldo de cien pesos mensuales, para que pueda atender y llenar los "grandes gastos" que esta importantísima comisión debe ocasionarle. ${ }^{24}$

La relación del Gobierno con la Liga es activa, esto es, no se trata de una administración que no opera políticamente hasta el advenimiento de un Poder Ejecutivo designado por la voluntad del voto de los diputados del Congreso provincial. Dos ejemplos pueden servirnos de muestra. 
La primera muestra se puede formular en tono de pregunta: ¿cómo contribuir con recursos en la Confederación con realidades provinciales desiguales? Esta cuestión se presenta a la hora de accionar en conjunto. Un buen ejemplo es qué hacer en caso de guerra; para decirlo de otro modo, en dicha situación todas las provincias deben colaborar del mismo modo o bien se negocia cómo debe hacerlo cada una. ¿Puede contribuir una provincia como Entre Ríos, con una situación económica grave en la que, durante la década de 1820, en ocasiones se rebajan los salarios e incluso le cuesta pagarlos? En un artículo de carácter reservado se puede notar a qué tipo de solución llegaron las provincias miembros:

Siendo notorio a todos los Gobiernos de la Liga, que los de Santa Fe y Entre Ríos no pueden por ahora en manera alguna
hacer frente a los gastos de la guerra, toda vez que ella sea necesaria, ambos Gobiernos quedan obligados a contribuir con sus
respectivos contingentes, según lo establecido en el artículo 13 del Tratado Publico, celebrado en esta ciudad de Santa Fe, y
en este día entre las tres provincias litorales, Santa Fe, Buenos Aires y Entre Ríos, y el gobierno de Buenos Aires se obliga a
propiciarles cuantos recursos pecuniarios le sean favorables, según sus atenciones y circunstancias para fomentar el apresto y
equipo de la fuerza con que cada uno de ellos deba contribuir, conforme a la designación del contingente que previamente
haya hecho la comisión representativa de los tres gobiernos litorales. ${ }^{25}$

Lo que observamos en este punto es que negocian cómo colaborar en caso de guerra. La resolución a la que llegan es que Buenos Aires lo hace con recursos económicos y Santa Fe y Entre Ríos, con recursos humanos. Todas las partes deben ceder algo a las otras y esforzarse por hacer algo que otros no harán. Entre Ríos y Santa Fe obtienen no contribuir con lo que no tienen, dinero, pero deben realizar el esfuerzo de proporcionar hombres para la guerra. Buenos Aires consigue no compartir el esfuerzo humano en la guerra, pero realiza el esfuerzo de contribuir más en recursos económicos. El hecho, por cierto, muestra cómo las partes que forman la Confederación buscan alternativas frente a situaciones en las que, efectivamente, no son iguales.

Una segunda cuestión puede advertirse en relación con el envío de diplomáticos en el marco del Pacto Federal. Una comunicación entre sus miembros nos hace ver cuál es el cariz de este problema. En abril de 1831, el gobernador delegado de Entre Ríos, José Ignacio Vera, le escribe al presidente de la comisión representativa que el presidente del Estado Oriental ha enviado a Evaristo Carriego en el "carácter de agente político cerca de este gobierno y el de Corrientes”. El episodio genera un punto novedoso, ya que el Pacto Federal modifica al respecto la posición que debe adoptar la provincia. En efecto, no es Entre Ríos la que decide por sí sola, sino que la Liga tiene la última palabra.

Por este motivo, el gobernador delegado en su comunicación da por sentado que, por el "artículo 4" de los Tratados celebrados en enero, "no puede el que firma ni oír, ni hacer proposiciones con otros gobiernos sin previo avenimiento expreso de las provincias que forman la presente federación". En este sentido, espera que los diputados de la Comisión Representativa puedan señalarle la "línea de conducta que en las circunstancias sea más prudente seguir, y más conforme con los intereses generales de la Liga” ${ }^{26}$

Con motivo de la nueva invasión de los unitarios, la Liga decide realizar un reclamo a las autoridades de la Banda Oriental. En mayo de 1831, el Gobierno de Buenos Aires autoriza al gobierno de Entre Ríos para que

pida del modo que estime conveniente al sor presidente del Estado Oriental del Uruguay explicaciones sobre haber permitido
que desde el territorio de su mando haya sido dos veces invadida esa misma provincia por varios emigrados de la República
Argentina y que exija que dichos emigrados sean alejados de las costas del Uruguay y vigilados en los puntos que residan, tiene
el honor de poner en su conocimiento que ha dirigido al Exmo Gobierno del Estado Oriental del Uruguay la reclamación
que en copia autorizada se acompaña.....

En el mes de julio, el gobernador delegado de Buenos Aires le solicita a su par entrerriano que, con el “objeto de dictar por su parte aquellas medidas que sean más conducentes a afianzar la quietud y tranquilidad de la Republica y con especialidad de las tres Provincias Federadas", se

sirva pasar una relación circunstanciada y prolija de los oficiales emigrados de la república que hayan tenido parte activa en las dos últimas invasiones hechas desde el Estado Oriental del Uruguay a esa provincia como igualmente de cualesquiera otros individuos de que tenga conocimiento que hayan cooperado bajo cualquier aspecto a la perpetración de aquellos atentados, 
y expresando si fuese posible en cuál de las invasiones hubiesen tomado parte o prestado su cooperación o si lo han hecho en las dos, y con qué clase de servicio ha concurrido cada uno por su parte. ${ }^{28}$

En suma, se advierte aquí cómo se pone en funcionamiento una red de comunicación y de estrategia común entre las provincias de la Confederación. Hacia fines de 1830 y 1831 se ve una acción más coordinada por parte de la Liga Federal. También es de notar la actitud dominante de Buenos Aires. El gobierno de Entre Ríos no parece una administración inactiva o ineficaz; lo que se nota es el intento ser una provincia miembro de la Liga que cumple o negocia cuestiones que se presentan para resolver.

\section{SITUACIÓN DE CONSPIRACIÓN Y PEDIDOS A LA COMISIÓN REPRESENTATIVA}

Más arriba señalamos cómo se produce una nueva invasión a la provincia en los primeros meses de 1831, sobre un escenario, en efecto, profundamente alterado. No es extraño, entonces, que lo primero a destacar sea el pedido, en diciembre de 1830, de facultades especiales solicitadas por el gobernador provisional. La solicitud es habitual en este tipo de cuadro histórico; lo que interesa enfatizar es qué tono discursivo emplea y, sobre todo, cuál es el objetivo de su pedido.

En su mensaje, el gobernador provisional traza en primer término un contexto particular. En las "críticas y espinosas circunstancias" que se viven, el objetivo básico es la "destrucción de los malvados", con la intención de evitar los "males inmensos" y proteger a la "infortunada patria"; en este marco, se admiten, pero también se solicitan a todos los habitantes, los "sacrificios" que la situación demanda. De esta manera, emerge una realidad particular que sale de lo habitual al presentarse "ocurrencias extraordinarias". El tiempo histórico se acelera vertiginosamente y por este motivo, es "preciso obrar con rapidez", en cuanto las circunstancias “imperiosamente [lo] exigen". Toda esta cadena de sentido, rodeada y atravesada por argumentos racionales, expresiones que apelan a la emoción y una retórica cerradamente dramática, lleva a la búsqueda de recursos para gastos de orden y de seguridad.

Los "gastos" que se reclaman remiten a los "multiplicados chasques, espías, vichadores", absolutamente necesarios, se afirma, para "salvar al país de la desolación y exterminio total que lo amenazan”. Por este motivo, el gobierno reclama al Congreso provincial "se le faculte amplia y extraordinariamente para obrar en las actuales circunstancias", pero también en "cuantas puedan ocurrir según el orden de los sucesos".

Una vez calmadas "las agitaciones y cesando lo extraordinario de las circunstancias", el Gobierno dará cuenta al Congreso provincial de "todas sus operaciones". El objetivo final es "la destrucción de los tiranos, salvación del país, y cimentar en él a toda costa el augusto sistema de los pueblos". 29

El Gobierno, como en el caso de la ley sobre el comercio que reconoce la figura del delator, aquí admite como otros empleos del Estado el trabajo de espías y otros infiltrados en el enemigo.

Se cree que la acción política del gobernador permite dar una respuesta a los movimientos conspirativos. De este modo, la acción política, como señala Lordon, es un asunto de afectos y deseos colectivos (Lordon, 2018, p. 17). La acción de los actores se mezcla con la actividad institucional: no son sujetos que actúan aislada o individualmente, sino que lo hacen dentro de una "estructura". En este cuadro de situación, actores e institución realizan tareas de reciprocidad. Por este motivo, justamente a ellos, actores e instituciones, apela cuando pide las facultades especiales, que se supone es el instrumento de poder adecuado para dar una solución.

Entre enero y febrero hay distintas comunicaciones entre miembros de la Liga sobre el conflicto latente en Entre Ríos. Hacia fines del primer mes, López le advierte a Rosas, por noticias que tiene de Barrenechea sobre lo que "piensa del presidente Rivera", de la necesidad "sobre la organización de los 300 hombres". En este sentido, afirma el gobernador de Santa Fe, "yo desearía” que si le es posible "enviase vestuario y algún armamento para ellos". ${ }^{30}$ 
El 12 de febrero, el Gobierno, con el objetivo de conservar el orden y la tranquilidad pública, "faculta al Sor. Alcalde Mayor para que sostenga una partida de diez hombres", quienes estarán al mando de "un sargento para cuyo servicio proporcionarán por su orden los gastos los haberes de la compañía y su manutención y demás necesarias". ${ }^{31}$

Los días 22, 23 y 24 de febrero, los acontecimientos se viven en un tiempo que se acelera, las comunicaciones adquieren un tono dramático, se adjuntan partes y otros documentos como demostración de lo que se describe, se recuerda la importancia de la causa política de la Liga; esto es, se ponen en obra distintos órdenes de argumentos y material documental.

El primer día mencionado, el gobernador de Entre Ríos le comunica al presidente de la Comisión Representativa la situación de su provincia con motivo de las sublevaciones recientes y pide auxilio. Le reclama que solicite al gobernador de Santa Fe "preste el auxilio, cuando menos, de cincuenta tercerolas y otros tantos sables con calidad de reintegro a la llegada del buque que de la capital de Buenos aires conduce estos artículos de guerra para el Entre Ríos". El objetivo es equipar a la "tropa veterana organizada" para que pueda salir a

dispersar las reuniones de anarquistas que en distintos puntos se dejan sentir en la provincia esperando al caudillo Ricardo López Jordán, quien según noticias positivas intenta nuevamente repasar el Uruguay, y causando males incalculables a todo el vecindario, tanto en los pueblos como en la campaña en donde por desgracia han principiado ya los cabecillas de esos grupos sublevados a cometer los mayores atentados, robando y fusilando hombres a la sombra de la impunidad en que se consideran al abrigo de los montes y de la inacción en que al gobierno le es preciso permanecer por falta de elementos. El pueblo de Gualeguay se encuentra circundado por las partidas de los sublevados, y amenazados los de Gualeguaychú y Uruguay porque todos se encuentran indefensos por la falta de armas. ${ }^{32}$

De no poder auxiliarla, señala que tenga en cuenta el "riesgo que corre su existencia política", pero también advierte sobre "la suerte y felicidad de esta provincia aliada". ${ }^{33}$

El 23 de febrero, el gobernador de Entre Ríos le solicita al presidente de la Comisión Representativa auxilio para las tropas en atención a los movimientos de Ricardo López Jordán, quien, "después de haber repasado el Uruguay", se dispuso a atacar al "Sor. Gral. Espino, y al Capitán Hereñú"; al mismo tiempo, señala que "uno de los que con él repasaron el Uruguay se halla con una partida fuerte en las inmediaciones de Nogoyá”.

Estos movimientos están señalados en el parte que adjunta. En este sentido, le señala la estrategia guerrera a seguir y la ayuda específica que necesita "el auxilio de cien hombres, suficientes" para que puedan ponerse en "unión de la gente que hay en esta". En este punto, aclara que en ellos "no puede tenerse mayor confianza (en) salir a batir al enemigo con el fin de no darle tiempo a conmover nuevamente la provincia y ponernos en los conflictos (anteriores)". Posteriormente, una vez más señala el peligro de la existencia de la provincia y su posición como provincia aliada. Esta vez también menciona que los auxilios son para "sostener los derechos de la Liga, y la causa sagrada de los pueblos". ${ }^{34}$

El 24 de febrero envía una nueva comunicación, acompañada de documentos, y pide auxilio con motivo de la "invasión de López Jordán, Urquiza y otros". Los documentos expresan concretamente que los mencionados "invasores" han "repasado el Uruguay con fuerza armada y se hallan en la provincia”. Luego, aclara que "esto mismo se sabe con más extensión por los informes verbales que el Sor. Gral. Espino ha encargado se den al infrascripto por los oficiales que conducen las comunicaciones”. Por estas razones, el gobernador señala que autoriza al diputado de la provincia para que explique esta grave situación y para que "sus aliados", los gobiernos de Santa Fe y Buenos Aires, envíen los auxilios solicitados. ${ }^{35}$

Unos días después se conoce la respuesta de Juan Manuel de Rosas, al comunicarse con el presidente de la Comisión Representativa sobre los auxilios para Entre Ríos. Califica los nuevos “disturbios” de Entre Ríos como "domésticos". Le informa sobre el "envío de 400 a 500 hombres de caballería a esa de Santa Fe", con el fin de auxiliar a Entre Ríos en caso necesario. Al mismo tiempo, señala que Buenos Aires ocupa el rol de "reserva" y que actuará "si el deber, las atenciones y la oportunidad estuviesen de acuerdo". ${ }^{36}$ Como se ve, 
las que aportan el principal recurso humano son Santa Fe y Entre Ríos, y Buenos Aires lo hace con recursos económicos.

En el mes de marzo, las cosas no parecen haberse modificado demasiado. Que esto es así lo muestra la nueva catarata de comunicaciones enviadas desde Entre Ríos. El primero de ese mes, aquel Gobierno le comunica al presidente de la Comisión Representativa que saldrá el secretario del general Espino para dar explicaciones de "alta importancia", con la intención de obtener un "auxilio suficiente". Todo ello es necesario en la "mayor brevedad para salvar aquella fuerza, y destrozar al enemigo que hoy aún no se halla en un estado demasiado fuerte". Y nuevamente se reitera que el fin último es la defensa que la "causa de los Pueblos demanda, la cual peligra sin el auxilio predicho". ${ }^{37}$

Una vez más, el 10 de marzo, el gobernador delegado de la provincia de Entre Ríos, José Ignacio de Vera, y su secretario, Calixto Vera, le comunican al presidente de la Comisión Representativa que es urgente el auxilio de caballería que ha solicitado. Es interesante, como se ha sostenido ya, cómo la solicitud siempre va acompañada de algún documento que testimonia lo que se describe:

el infrascripto protesta al Sr Presidente a que se dirige que nunca es más urgente el auxilio solicitado que en las presentes circunstancias, como puede imponerse por la carta original del Sor. gobernador en campaña, que se ha remitido al Sor. diputado de la misma comisión D. Domingo Cullen y el que firma espera que el Sr. presidente se empeñará más y más en que se apresure el expresado auxilio, por el peligro que existe en que los triunfos conseguidos, se pierdan, a causa de los motivos que se indican en la carta referida. ${ }^{38}$

En suma, los auxilios van llegando, pero no en los tiempos en que se los solicita. El conflicto abierto provoca que las comunicaciones alcancen un tono dramático, tanto para la confrontación abierta como para su conclusión. La resolución de las hostilidades se da en el terreno de la negociación pacífica. Un tratado, finalmente, es firmado en el Arroyo del Ceibo el 22 de marzo de 1831. Rápidamente fue ratificado por las partes. ${ }^{39}$

Para tal convenio, el General en Jefe del Ejército de los Libres nombra como representantes a D. Manuel Bustos y D. Martiniano Chilavert (quien ya conspiró en la revolución de 1830), y el Inspector General de Armas de la Provincia de Entre Ríos designa al Sargento Mayor D. Juan Manuel Aldao. El objetivo es ajustar un Tratado definitivo en el que se arreglen los intereses de las partes beligerantes. A modo de "garantía" interviene a nombre del Excmo. Gobierno de Corrientes el Teniente Coronel Jefe de la fuerza auxiliar, por medio del comisionado al efecto Teniente Coronel D. Manuel José Fernández después de haber canjeado sus respectivos poderes han convenido en los artículos siguientes.

El artículo 1 dispone que "todas las personas que hayan pertenecido al partido denominado Unitario y pertenezcan a la Provincia de Entre Ríos serán respetadas en su individuo e intereses”. El artículo 2 señala que "desde la ratificación de este tratado habrá un perpetuo olvido sobre todos los acontecimientos anteriores". En el artículo 3 se afirma que "las fuerzas pertenecientes al Ejército de los Libres se retirarán a sus respectivos Departamentos con sus armas", pero se aclara que deben "entregarlas en el término de doce días en el punto que el gobierno estimare conveniente". En el artículo 4 se afirma que "a consecuencia de este tratado el Señor General D. Juan Lavalle repasará el Uruguay con su fuerza, armas, y caballos, en el término de cuarenta y ocho otras". En el artículo 5 se sostiene que "las fuerzas auxiliares del Gobierno de Corrientes deberán existir en la provincia hasta el cumplimiento del artículo $3^{\circ}$ de este tratado", el cual será "ratificado por los Jefes de ambas fuerzas".

La negociación de paz cierra el conflicto. Sin embargo, el orden en la provincia aún se deja oír los reclamos sobre falta de orden y de seguridad de sus habitantes. A fines de marzo, el gobernador le informa al alcalde mayor y comandante político, Domingo Calvo, que, si bien lo pone en contacto con el Inspector General de Armas para la protección de un pueblo que la solicita, "no puede asegurar su ida a ese Pueblo por cuanto ignora si las circunstancias se lo permitirán”. ${ }^{40} \mathrm{El}$ hecho muestra la incertidumbre, la falta de un escenario seguro y la imposibilidad del primer mandatario de garantizar con su presencia una solución. 
Algunos indicios, al mismo tiempo, nos señalan que el Gobierno se hizo cargo con recursos de estos destinos públicos y su sustento. En el mes de abril, desde un campamento militar se señala que "queda recibido de los trecientos pesos o pagos que usted ha servido franquearme para el socorro de las tropas de mi mando y a más de tributar(...)en obsequio de tantos abultados servicios protejo devolver a v. dicha suma contenida en la misma moneda". ${ }^{41}$

\section{Gobierno de "extraordinarias circunstancias": un mecanismo de crisis y de descontrol, frontera de actores móviles}

Intenté mostrar a lo largo de este trabajo que el caso del Gobierno provisional de Barrenechea no se ajusta a la idea de una administración inactiva y sin propuesta ante las dificultades que presenta la escena entrerriana de los años de 1830. Por el contrario, es una administración con múltiples iniciativas de gobierno y con tomas de decisiones en áreas de poder. Podemos separar sus iniciativas en dos partes. Presenta, por un lado, iniciativas conocidas en la política entrerriana (renovación del plantel de empleos y cargos, órdenes para recaudar más y mejor, no gastar más de lo que el erario puede solventar, etc.). Sin embargo, promociona, por otro lado, medidas novedosas: reestructura el mando militar y judicial de poder en la provincia, con la finalidad de dar una solución definitiva al problema de la falta de autoridad y a los permanentes intentos de golpes de Estado. Todo ello se da en un marco que repiensa la administración provincial y su estatuto constitucional.

Se puede conjeturar, a modo de hipótesis, que el esfuerzo de gestión y estrategia por parte de Barrenechea responde a dos órdenes de objetivos: por un lado, cortar en seco el viejo problema del desorden intentando crear un gobierno de estabilidad, que trata particularmente de superar el problema de las dos Comandancias militares; y, por otro lado, a partir de las expectativas de que las medidas implementadas logren éxito, imponerse como gobernado propietario.

Esa abundancia de iniciativas y de propuestas de cambios, sin embargo, no pudo dar respuestas a lo que he denominado mecanismo de crisis y de descontrol, ya que el cielo del breve período gubernamental de Barrenechea nunca o casi nunca parece brillar sin nubes: y fue necesariamente de este modo, si tenemos en cuenta que siguieron sucediéndose distintos órdenes de problemas; amenazas e invasiones a la provincia, anormalidad institucional, dificultades en el erario.

En este clima de inestabilidad conspirativa casi permanente, dentro del campo del juego de la política de los actores traté de mostrar que se trazaron una suerte de fronteras de actores móviles que permitieron observar sus movimientos: si terminada la intentona revolucionaria del mes de diciembre, el "nosotros" incluía a las fuerzas del flamante Gobierno provisional apoyadas por la Liga Federal y el "ellos" comprendía a los vencidos sublevados, eso se modifica a las pocas semanas, cuando comienza la invasión posterior del "partido unitario" de Lavalle, López Jordán y los hermanos Urquiza; vencidos, el “ellos” se traslada hacia adentro de la administración entrerriana con dos potenciales adversarios, el ministro de Gobierno Vera y el Inspector de Armas, Espino, quien finalmente desplaza a Barrenechea en julio.

Ahora bien, ese Gobierno provisional que puso en obra varias iniciativas de modo simultáneo y que atendió actividades de invasión y conflictos bélicos, ¿por qué fracasa? Bien podría decirse, sólo a modo de conjetura, que no logra contar con un actor o actores que sostengan tanto sus iniciativas como sus reformas. De este modo, no logra el éxito esperado, pero no por falta de iniciativa o por inercia. El arte de la política de articular las partes del cuadro de todos los sectores (élite, sectores populares, etc.) es lo que falla (y muy claramente, por cierto). Si bien en este trabajo no me detuve a examinarlo y sólo hay aquí alguna referencia, no es exagerado suponer lo que afirmamos si tomamos en cuenta que no hay sectores que salgan a la luz pública a defender a Barrenechea en momentos de debilidad, salvo cuando aparece la invasión del "partido unitario". 


\section{REFERENCIAS}

Bosch, B. (1991). Historia de Entre Ríos. Buenos Aires: Plus Ultra.

Bourdieu, P. (1997). Razones prácticas. Sobre la teoría de la acción. Barcelona: Anagrama.

Fradkin, R. (2006). La historia de una montonera. Bandolerismo y caudillismo en Buenos Aires, 1826. Buenos Aires: Siglo Veintiuno editores.

Herrero, F. (2007). La difícil construcción de una institución republicana. La Sala de Representantes durante el gobierno de José María Paz. Córdoba, hacia 1830, Anuario del IEHS, 22, 41-69. Recuperado de http://anuarioiehs.unicen.edu.ar/Files/2007/Ficci\%C3\%B3n\%20legal,\%20iniciativa\%20seria \%20y\%20exitosa\%20o\%20intento\%20que\%20presenta\%20dificultades.\%20Una\%20aproximaci\%C3\%B3n \%20al\%20estudio\%20de\%20la\%20Sala\%20de\%20Representantes,\%20C\%C3\%B3rdoba,\%201829-1831..pdf

Lordon, F. (2018). La sociedad de los afectos. Por un estructuralismo de las pasiones. Buenos Aires: Adriana Hidalgo editora.

Martínez, B. (1910). Historia de Entre Ríos. Buenos Aires: Editorial Graf. de Rosso y cía.

Pressel, G. (2020). Territorio y configuración judicial a mediados del siglo XIX. Comandantes y prácticas judiciales en la campaña entrerriana. En F. Herrero y G. Pressel. Entre Rios, siglo XIX. Lenguajes y prácticas, en un imaginario politico dinámico y cambiante. (En Prensa). Paraná: Editorial UADER.

Tedeschi, S. (2020). Las finanzas públicas en tiempos de transformación política. Entre Ríos 1820-1832. En F. Herrero y G. Pressel. Entre Rios, siglo XIX. Lenguajes y prácticas, en un imaginario político dinámico y cambiante. (En Prensa). Paraná: Editorial UADER.

Tío Vallejo, G. (2011). La República extraordinaria. Tucumán en la primera mitad del siglo XIX. Rosario: Prohistoria. Todd, A. (2000). Las revoluciones, 1789-1917. Madrid: Alianza Editores.

\section{FUENTES DOCUMENTALES UTILIZADAS}

Fondo General Justo José de Urquiza, correspondencia, 1830-1835. Archivo General de la Nación (AGN), Sala VII, legajo 1464 .

Fondo Gobierno, Serie III, Tratados Interprovinciales 1824-1852. Archivo Histórico de Entre Ríos (AHER), Caja 1, 1820-1852. Legajo 12, año 1831.

Documentos para la Historia Argentina. Relaciones interprovinciales. La Liga del Litoral (1829-1833) (DHA-RILL). Tomo XV, Buenos Aires, 1922.

Recopilación de leyes, decretos y acuerdos de la Provincia de Entre Ríos, desde 1821 a 1873 (RLDER). Tomo III (1821-1832), Uruguay, Imprenta La Voz del Pueblo, 1875.

\section{ANEXO}

\section{Cuadro 1. Gobernadores de Entre Ríos}

\section{Designaciones del Directorio}

Gobernadores período

Blas de Pico 10-9-1814 /4-11-1814 Interino desde el 6 de agosto de 1814, previo al decreto de su creación Juan José Viamonte 4-11-1814/16-1-1815

Eusebio Valdenegro 16-1-1815 abandonó provincia, febrero de 1815

Bajo el signo del artiguismo 
Eusebio Hereñú 1816/setiembre 1817

José Ignacio Vera set-1817/26-3-1818

José Francisco Rodríguez 26-3-1818/23-2-1820 bajo protección de Artigas y Ramírez

Período Tratado de Pilar

Francisco Ramírez 23-2-1820/29-9-1820

Período República de Entre Ríos

Francisco Ramírez 29-9-1820/10-7-1821 Jefe Supremo de la República de ER Ricardo López Jordán 10-7-1821/28-9-1821 Interino

En el marco general del período de la Constitución provisoria

Lucio Mansilla 28-9-1821/13-12-1821 Provisional, derrocó a López Jordán

Lucio Mansilla 13-12-1821/ 12-2-1824

Juan León Sola 12-2-1824/4-4-1826

Vicente Zapata 4-4-1826/1-3-1827 Provisional

Mateo García de Zúñiga 13-3-1827/29-9-1827 [falta el mes]

Vicente Zapata 29-9-1827/16-12-1827 Provisional

Pedro Pablo Seguí 16-12-1827/19-12-1829

Juan León Sola 19-12-1829/1-1-1830 Provisional

Juan León Sola 1-1-1830/16-11-1830

Pedro Barrenechea 16-11-1830/22-11-1830 Provisional

Ricardo López Jordán 22-11-1830/10-12-1830 Provisional

Pedro Barrenechea 10-12-1830/11-7-1831 Provisional [¿ंSin fecha precisa?

Pedro Espino 11-7-1831/14-12-1831

Valentín Denis 14-12-1831/17-12-1831 Pte. de la As. Gral. Extr

Toribio Ortiz 17-12-1831/1-3-1832

Pascual Echague 1-3-1832/31-12-1841

Vicente Zapata 31-12-1841/1-1-1842 Provisional

Justo José Urquiza 1-1-1842/29-1-1842

Pedro Pablo Seguí 29-1-1842/12-3-1842 Provisional

José María Paz 12-3-1842/3-4-1842

Francisco Dionisio Álvarez 3-4-1842/7-4-1842

Justo José de Urquiza 7-4-1842/24-3-1854

Fuente: Antonio Zinny, Historia de los gobernadores de las provincias argentinas desde 1810 hasta la fecha: Provincias litorales, C. Casavalle, 1879. (Elaboración)

\section{Notas}

1 La inestabilidad continuó hasta que, en marzo del año siguiente, se hizo cargo del Gobierno el exmMinistro de Gobierno santafesino Pascual Echagüe. Éste nombró a Barrenechea comandante del departamento de Paraná, cargo que ocupó hasta fines de 1833. Fue su última actuación pública.

2 “Comunicación”, Paraná, 5-7-1831, Recopilación de leyes, decretos y acuerdos de la Provincia de Entre Ríos desde 1821 a 1873 (en adelante, RLDER). Tomo III (1821-1832), Concepción del Uruguay Uruguay, Imprenta La Voz del Pueblo, 1875 , p. 308.

3 “Comunicación”, Paraná, 6-7-1831, RLDER. T.III, p. 309. 
4 "Mensaje del Gobierno a la Legislatura. Sobre conveniencia de suspender nombramiento de electores". Paraná, 11-12-1830, RLDER. T.III, p. 229.

5 “Acuerdo. Poder Ejecutivo”, Paraná, 14-1-1831, RLDER. T.III, p. 259.

6 “Acuerdo. Sobre reposición de empleo”, Paraná, 11-12-1830, RLDER. T.III, p. 230.

7 “Acuerdo. Poder Ejecutivo”, Paraná, 14-1-1831, RLDER. T.III, pp. 262-263.

8 “Decreto”, Paraná, 17-1-1831, RLDER. T.III, pp. 265-266.

9 “Acuerdo. Nombra Comandantes”. Paraná, 31-12-1830, RLDER. T.III, p. 239.

10 “Acuerdo. Nombra Comandantes”. Paraná, 31-12-1830, RLDER. T.III, p. 239.

11 “Acuerdo. Sobre pagos al Estado”. Paraná, 17-12-1830, RLDER. T.III, p. 233.

12 “Acuerdo. Sobre el pago de derechos de exportación”. Paraná, 20-12-1830, RLDER. T.III, p. 234.

13 “Acuerdo. Poder Ejecutivo”, Paraná, 3-1-1831, RLDER. T.III, p. 242.

14 “Pedro Barrenechea a Del castillo", Paraná, 8-2-1831.

15 “Acuerdo. Sobre venta de sellos para licencias de embarque”. Paraná, 15-12-1830, RLDER. T.III, p. 232.

16 Idem

17 “Decreto”, Paraná, 17-1-1831, RLDER. T.III, p.p. 269-270.

18 "Pedro Barrenechea a Del castillo", Paraná, 9-2-1831.

19 “Congreso Provincial al Gobernador”, Paraná, 17 y 18-1-1831. Sala VII, Fondo General Justo José de Urquiza. Legajo 1464, correspondencia, 1830-1835, Archivo General de la Nación (en adelante, AGN).

20 “Congreso Provincial al Gobernador”, Paraná, 17 y 18-1-1831. Sala VII, Fondo General Justo José de Urquiza. Legajo 1464, correspondencia, 1830-1835, AGN.

21 “Congreso Provincial al Gobernador”, Paraná, 19-1-1831, Sala VII, Fondo General Justo José de Urquiza. Legajo 1464, correspondencia, 1830-1835, AGN.

22 “Mensaje”, Paraná, 20-1-1831, RLDER. T.III, p. 275.

23 “Orden”, Paraná, 20-1-1831, RLDER. T.III, p. 273. El gobierno manda al alcalde mayor para que la publique en bando y se dé a conocer la ley. "Circular”, Pedro Barrenechea, Paraná, 31-1-1831.

24 “Acuerdo. Nombran diputados para la Liga”. Paraná, 31-12-1830, RLDER. T.III, pp. 237- 238.

25 "Articulo reservado adicionado del Tratado de 4 de enero celebrado entre las provincias litorales el año 1831". Sala VII, Fondo General Justo José de Urquiza. Legajo 1464, correspondencia, 1830-1835, AGN.

26 “Gobernador delegado de Entre Ríos al Presidente Comisión Representativa”, Paraná, 20 abril 1831, DHA-RILL, T.XVI, p. 266.

27 "Gobierno de Buenos Aires al Gobernador delegado de Entre Ríos". 5-5-1831, DHA-RILL, T.XV, p. 124. Sobre el mismo reclamo, véase "Gobierno de Buenos Aires al Gobernador delegado de Santa Fe", 5-5-1831, DHA-RILL. T.XV, pp. 125-126.

28 "Gobierno de Buenos Aires al Gobernador delegado de Entre Ríos". 5-7-1831, DHA-RILL, T.XV, pp. 128-129.

29 “Mensaje del gobierno. Sobre facultades extraordinarias". Paraná, 23-12-1830, RLDER. T.III, pp. $234-236$.

30 “Carta de López a Rosas”, Santa Fe, 26 enero 1831, Correspondencia...ob. Cit. pp. 131-132.

31 "Circular", 12-2-1831, firma Barrenechea.

32 “Gobernador Entre Ríos al Presidente Comisión Representativa”, Paraná, 22 febrero de 1831, DHA-RILL, T.XVI, p. 239.

33 Idem

34 “Gobernador Entre Ríos al Presidente Comisión Representativa”, Paraná, 23 de febrero 1831, DHA-RILL, T.XVI, p. 243.

35 “Gobernador Entre Ríos al Presidente Comisión Representativa”, Paraná, 24 de febrero 1831, DHA-RILL, T.XVI, p. 244.

36 "Gobernador de Buenos Aires al Presidente Comisión Representativa”, Buenos Aires, 27 de febrero de 1831, DHARILL, T.XVI, pp. 245-246.

37 "Gobernador de Entre Ríos al Presidente Comisión Representativa”, Paraná, 1 de marzo 1831, DHA.RILL. T.XVI, p. 247.

38 "Gobernador delegado de Entre Ríos al Presidente Comisión Representativa”, Paraná, 10 marzo 1831, DHA.RILL. T.XVI, p. 257.

39 “Convenio con el General Lavalle y la provincia de Entre Ríos”, Arroyo del Ceibo, 22-3-1831. Fondo Gobierno, Serie III, Tratados Interprovinciales, 1824-1852. Caja 1, 1820-1852. Legajo $\mathrm{N}^{\circ} 12$, año 1831, Archivo Histórico de Entre Ríos.

40 "Pedro Barrenechea a Domingo Calvo alcalde mayor y comandante político", cuartel general, 25-3-1831.

41 “José López a José Viezo", campamento en Mandisoví,7-4-1831. [La grafía actual es "Mandisoví". La de la época ¿era sin tilde?] 